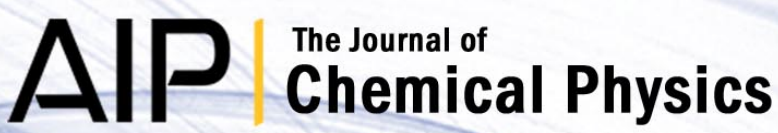

Accurate quantum-mechanical rate constants for a linear response AzzouzBorgis proton transfer model employing the multilayer multiconfiguration time-dependent Hartree approach

Ian R. Craig, Michael Thoss, and Haobin Wang

Citation: J. Chem. Phys. 135, 064504 (2011); doi: 10.1063/1.3624342

View online: http://dx.doi.org/10.1063/1.3624342

View Table of Contents: http://jcp.aip.org/resource/1/JCPSA6/v135/i6

Published by the American Institute of Physics.

Additional information on J. Chem. Phys.

Journal Homepage: http://jcp.aip.org/

Journal Information: http://jcp.aip.org/about/about_the_journal

Top downloads: http://jcp.aip.org/features/most_downloaded

Information for Authors: http://jcp.aip.org/authors

\section{ADVERTISEMENT}

\section{ACCELERATE COMPUTATIONAL CHEMISTRY BY 5X. TRY IT ON A FREE, REMOTELY-HOSTED CLUSTER. \\ RVIDIA.}




\title{
Accurate quantum-mechanical rate constants for a linear response Azzouz-Borgis proton transfer model employing the multilayer multiconfiguration time-dependent Hartree approach
}

\author{
Ian R. Craig, ${ }^{1, a)}$ Michael Thoss, ${ }^{2, b)}$ and Haobin Wang ${ }^{3,4, b)}$ \\ ${ }^{1}$ Theoretische Chemie, Department Chemie, Technische Universität München, 85748 Garching, Germany \\ ${ }^{2}$ Institut für Theoretische Physik und Interdisziplinäres Zentrum für Molekulare Materialien, \\ Friedrich-Alexander-Universität Erlangen-Nürnberg, Staudtstr. 7/B2, D-91058 Erlangen, Germany \\ ${ }^{3}$ Department of Chemistry and Biochemistry, MSC 3C, New Mexico State University, Las Cruces, \\ New Mexico 88003, USA \\ ${ }^{4}$ Beijing Computational Science Research Center, No. 3 He-Qing Road, Hai-Dian District, Beijing 100084, \\ People's Republic of China
}

(Received 5 July 2011; accepted 19 July 2011; published online 12 August 2011)

\begin{abstract}
The multilayer multiconfiguration time-dependent Hartree (ML-MCTDH) method is applied to simulate the quantum dynamics and thermal rate constant of the Azzouz-Borgis model of proton transfer in a polar solvent. To this end, the original atomistic potential is mapped to a system-bath model. Employing the flux correlation function formalism and importance sampling techniques, accurate quantum mechanical rate constants are obtained, which provide a benchmark for evaluating approximate approaches to study the quantum dynamics of condensed-phase chemical reactions. Furthermore, the validity of the mapping procedure is discussed based on the comparison of the classical dynamics of the original atomistic Azzouz-Borgis model and the mapped system-bath model. ( 2011 American Institute of Physics. [doi:10.1063/1.3624342]
\end{abstract}

\section{INTRODUCTION}

A central goal in chemical reaction dynamics is the accurate evaluation of thermal rate constants. Among various approaches, the reactive flux correlation function formalism ${ }^{1,2}$ offers a particularly efficient way to achieve this goal because it avoids the explicit calculation of state-to-state reaction probabilities. Nevertheless, at least two major difficulties arise when applying this formalism to chemical reactions which occur in condensed-phase environments. ${ }^{3}$ The first is that in order to evaluate the flux correlation function it is necessary to propagate the real-time quantum dynamics of the entire system. This is a demanding task for condensed-phase chemical reactions due to the involvement of a large number of coupled degrees of freedom. ${ }^{4-6} \mathrm{~A}$ second problem is that in a complex molecular system a large number of thermal flux eigenstates are in principle involved in determining the flux correlation function, ${ }^{3}$ which makes a direct evaluation computationally unfeasible.

The recently-proposed multilayer multiconfiguration time-dependent Hartree (ML-MCTDH) approach for evaluating reactive quantum dynamics seeks to alleviate both difficulties. ${ }^{3}$ Firstly, it employs the highly efficient ML-MCTDH wavepacket propagation algorithm ${ }^{7,8}$ in order to describe the quantum dynamics of many coupled degrees of freedom. Secondly, an importance sampling scheme is used to select those thermal flux eigenstates that make significant contributions to the flux correlation function. The approach

\footnotetext{
a)Present address: BASF SE, GVC/C - A030, D-67056 Ludwigshafen, Germany.

b) Authors to whom correspondence should be addressed. Electronic mail: michael.thoss@physik.uni-erlangen.de and whb@intrepid.nmsu.edu.
}

has been applied to two well-studied but relatively simple test models ${ }^{9}$ of condensed-phase proton transfer reactions and was shown to be an effective way to obtain accurate quantummechanical rate constants across a wide range of physical regimes. ${ }^{10}$

In this paper, we present an application of this methodology to the Azzouz-Borgis model of proton transfer in a polar solvent. ${ }^{11}$ The reasons for studying this particular model are twofold. Firstly, it provides a significantly more realistic model of a condensed-phase proton transfer process than those which were previously studied with the MLMCTDH approach. ${ }^{10}$ Secondly, although various approximate quantum-mechanical and quantum-classical methods have already been applied to this model, ${ }^{12-18}$ there is considerable disagreement between the resulting rate constants. By providing an accurate quantum-mechanical evaluation, this application of the ML-MCTDH approach to the AzzouzBorgis model may help in clarifying the reasons for the differences in the previously calculated rate constants.

The rigorous application of the ML-MCTDH method to reactive dynamics in systems with many degrees of freedom is currently limited to Hamiltonians of a particular form - a sum of products of single-particle operators. To apply the method to the Azzouz-Borgis model, we use a standard protocol that maps the complicated atomistic Hamiltonian within linear response onto a "system-bath" model. ${ }^{13,19-21}$ The latter is composed of a reaction coordinate of interest (the "system") coupled to a "bath" of harmonic oscillators and has the required product form. ${ }^{22,23}$

The paper is organized as follows. The ML-MCTDH approach to simulate flux correlation functions and thermal rate constants is summarized in Sec. II. Section III discusses the 
mapping of the original atomistic Azzous-Borgis model of proton transfer in a polar solvent to a system-bath model. Section IV presents the results of the ML-MCTDH calculations for the flux correlation functions and the thermal rate constant and gives a comparison with previous results of approximate theories. In addition, the accuracy and validity of the mapped version of the Azzouz-Borgis model is evaluated by comparing its classical dynamics and classical rate constants with those of the original atomistic Hamiltonian. Section V concludes with a summary.

\section{THE ML-MCTDH APPROACH TO CALCULATE THERMAL RATE CONSTANTS BASED ON THE FLUX-CORRELATION FUNCTION FORMALISM}

The combination of the flux-correlation function formalism with the capabilities of the ML-MCTDH method to accurately treat the quantum dynamics of systems with many degrees of freedom provides a numerically exact methodology to calculate thermal rate constants in complex molecular systems. The methodology and the details of implementation have been described in Refs. 3 and 10. Here we summarize the basic ideas and discuss some details specific to the application considered in this work.

As in our previous work, ${ }^{3,10}$ we adopt the following commonly-used definition of the flux correlation function, ${ }^{24}$

$$
C_{f}(t)=\operatorname{tr}\left[e^{-\beta \hat{H} / 2} \hat{F} e^{-\beta \hat{H} / 2} e^{+i \hat{H} t} \hat{h} e^{-i \hat{H} t}\right],
$$

where $\beta=1 / k_{B} T$ and $\hat{H}$ is the Hamiltonian. Throughout this article we use atomic units such that $\hbar=1$. In Eq. (2.1), the side operator $\hat{h}$ projects onto the product side of the chosen reaction dividing surface and the flux operator $\hat{F}$,

$$
\hat{F}=i[\hat{H}, \hat{h}],
$$

is the Heisenberg time-derivative of $\hat{h}$.

The flux correlation function is a valuable source of information on the dynamics of a reactive process. Its long-time limit determines the experimentally measured thermal reaction rate. Specifically, for a unimolecular chemical reaction occurring in a condensed-phase environment, the forward rate constant can be extracted from $C_{f}(t)$ as ${ }^{10}$

$$
k(T)=\frac{1}{Q_{r}(T)} \lim _{t \rightarrow \infty} \frac{C_{f}(t)}{P_{r}(0)+\left[P_{r}(0)-1\right]\left[Q_{r}(T) / Q_{p}(T)\right]-\left[Q_{r}(T)^{-1}+Q_{p}(T)^{-1}\right] \int_{0}^{t} d \tau C_{f}(\tau)} .
$$

Thereby, it is assumed that the long-time dynamics of the reactive system are governed by a rate process, i.e.,

$$
\lim _{t \rightarrow \infty} \frac{d}{d t} P_{r}(t)=-k(T) P_{r}(t)+k_{b}(T) P_{p}(t),
$$

and that the condensed-phase environment ensures that the system eventually reaches thermodynamic equilibrium, i.e.,

$$
\frac{k(T)}{k_{b}(T)}=\frac{Q_{p}(T)}{Q_{r}(T)} .
$$

In the preceding equations, $k_{b}(T)$ is the backward rate constant, $Q_{r}(T)$ and $Q_{p}(T)$ are the reactant and product partition functions,

$$
\begin{gathered}
Q_{r}(T)=\operatorname{tr}\left[e^{-\beta \hat{H}}(1-\hat{h})\right], \\
Q_{p}(T)=\operatorname{tr}\left[e^{-\beta \hat{H}} \hat{h}\right],
\end{gathered}
$$

and $P_{r}(t)$ and $P_{p}(t)$ are the time-dependent populations of the reactant and product regions of the system

$$
P_{r}(t)=\frac{1}{Q_{r}(T)} \operatorname{tr}\left[e^{-\beta \hat{H} / 2}(1-\hat{h}) e^{-\beta \hat{H} / 2} e^{i \hat{H} t}(1-\hat{h}) e^{-i \hat{H} t}\right],
$$

$$
P_{p}(t)=\frac{1}{Q_{r}(T)} \operatorname{tr}\left[e^{-\beta \hat{H} / 2}(1-\hat{h}) e^{-\beta \hat{H} / 2} e^{i \hat{H} t} \hat{h} e^{-i \hat{H} t}\right] .
$$

The expression for $k(T)$ that we have given here [Eq. (2.3)] was derived only recently, ${ }^{10}$ but it reduces to a more commonly-used form ${ }^{1,25}$ if two conditions are met. Firstly, there must be a significant separation between the timescale of the reaction $\left(\tau_{\mathrm{rc}}\right)$ and the timescales of the other dynamical processes in the system $\left(\tau_{\mathrm{mol}}\right)$. Then, at intermediate "plateau" times $t_{\mathrm{p}}$,

$$
\tau_{\mathrm{mol}} \ll t_{\mathrm{p}} \ll \tau_{\mathrm{rc}},
$$

there will have been negligible loss of reactant population ${ }^{26}$ whilst any transient structure from nonreactive molecular motion will have been quenched by decoherence. ${ }^{27,28}$ For such times the flux correlation function is independent of time and the previous expression for the rate constant (Eq. (2.3)) becomes

$$
k(T)=\frac{1}{Q_{r}(T)} \lim _{t \rightarrow t_{\mathrm{p}}} \frac{C_{f}(t)}{P_{r}(0)+\left[P_{r}(0)-1\right]\left[Q_{r}(T) / Q_{p}(T)\right]} .
$$

The second condition needed for the simplification of Eq. (2.3) is that the initial reactant population must be close to one $\left[P_{r}(0) \simeq 1\right]$. In this case it is clear that Eq. (2.3) reduces to

$$
k(T)=\frac{1}{Q_{r}(T)} \lim _{t \rightarrow \infty} \frac{C_{f}(t)}{1-\left[Q_{r}(T)^{-1}+Q_{p}(T)^{-1}\right] \int_{0}^{t} d \tau C_{f}(\tau)} .
$$

When both conditions hold, the appropriate expression is the commonly-used form ${ }^{1}$

$$
k(T)=\frac{1}{Q_{r}(T)} \lim _{t \rightarrow t_{\mathrm{p}}} C_{f}(t) .
$$

Whichever expression for $k(T)$ is appropriate [Eq. (2.3), Eq. (2.9), or Eq. (2.11)], it is first necessary to calculate the flux correlation function by evaluating the trace in Eq. (2.1). 
For this purpose, a convenient choice of basis is the set of eigenstates of the "Boltzmannized" flux operator, i.e.,

$$
\hat{F}_{\beta}\left|w_{n}\right\rangle=f_{n}\left|w_{n}\right\rangle
$$

where

$$
\hat{F}_{\beta}=e^{-\beta \hat{H} / 2} \hat{F} e^{-\beta \hat{H} / 2} .
$$

In this basis the flux correlation function is

$$
C_{f}(t)=\sum_{n} f_{n}\left\langle w_{n}(t)|\hat{h}| w_{n}(t)\right\rangle,
$$

where the time-evolved flux eigenstates are

$$
\left|w_{n}(t)\right\rangle \equiv e^{-i \hat{H} t}\left|w_{n}\right\rangle
$$

Considerable progress has been made over the last two decades in simulating reactive flux correlation functions for small gas phase reactions. The most significant methodological advances are based on the observation that the Boltzmannized flux operator $\hat{F}_{\beta}$ in Eq. (2.13) is of low rank. ${ }^{29-31}$ In particular, Manthe and co-workers ${ }^{32-34}$ have successfully developed MCTDH-based methods to evaluate flux correlation functions for various gas phase reactions. However, for chemical reactions occurring in condensed-phase systems the Boltzmannized flux operator is no longer of low rank. The number of eigenstates of $\hat{F}_{\beta}$ is so large that an evaluation of each term in the sum of Eq. (2.14) would be impractical. Furthermore, this direct summation of all flux eigenstates is unnecessary since some of them make insignificant contributions to $C_{f}(t)$.

Thus, rather than attempting an evaluation of Eq. (2.14), the ML-MCTDH-based approach proposed recently ${ }^{3,10}$ uses an importance sampling scheme in order to focus computational resources on the significant contributions to the flux correlation function. The first step is the definition of a "zeroth order" Hamiltonian

$$
\hat{H}_{0}=\hat{H}_{S}+\hat{H}_{B},
$$

in which the low-dimensionality "system" Hamiltonian $\left(\hat{H}_{S}\right)$ acts on different degrees of freedom than the Hamiltonian for the "bath" $\left(\hat{H}_{B}\right)$. Secondly, $\hat{H}_{0}$ is introduced into the flux correlation function using $\hat{1}=e^{-\beta \hat{H}_{0} / 2} e^{+\beta \hat{H}_{0} / 2}$ and the resulting expression is rearranged after requiring, without loss of generality, that $\hat{F}$ and $\hat{h}$ only operate on the system $\left(\hat{H}_{S}\right)$ degrees of freedom. Finally, the trace is evaluated in a product basis composed of the eigenstates of the system Boltzmannized flux operator

$$
e^{-\beta \hat{H}_{S} / 2} \hat{F} e^{-\beta \hat{H}_{S} / 2}\left|u_{n}\right\rangle=f_{n}\left|u_{n}\right\rangle,
$$

and the bath energy eigenstates

$$
\hat{H}_{B}|k\rangle=E_{k}|k\rangle \text {. }
$$

This leads to the final expression for $C_{f}(t)$ :

$C_{f}(t)=Q_{B}(T) \sum_{k} \frac{e^{-\beta E_{k}}}{Q_{B}(T)} \sum_{n} f_{n}\left\langle\Phi_{n, k}^{\beta}\left|e^{+i \hat{H} t} \hat{h} e^{-i \hat{H} t}\right| \Phi_{n, k}^{\beta}\right\rangle$,

where we have defined

$$
\left|\Phi_{n, k}^{\beta}\right\rangle \equiv e^{-\beta\left[\hat{H}-\left(\hat{H}_{S}+E_{k}\right)\right] / 2}\left|u_{n}\right\rangle|k\rangle,
$$

and have introduced the bath partition function

$$
Q_{B}(T)=\sum_{k} e^{-\beta E_{k}} .
$$

According to Eq. (2.19) each sampled initial state , $\left|u_{n}\right\rangle|k\rangle$, must be propagated in both real and imaginary time [Eqs. (2.19) and (2.20)] employing the of the fully-coupled Hamiltonian $\hat{H}$. Evaluating this time evolution is challenging for a chemical reaction occurring in a condensed-phase environment because a large number of coupled degrees of freedom are typically involved. ${ }^{4-6}$ The conventional approaches to calculating reactive quantum dynamics,${ }^{35}$ which were developed to treat small-molecule gas-phase reactions, are not feasible here. The ML-MCTDH approach provides a powerful alternative.

The ML-MCTDH theory ${ }^{7}$ is a rigorous variational method to propagate wave packets in complex systems with many degrees of freedom. In this approach, the wave function is represented by a recursive, layered expansion,

$$
\begin{aligned}
|\Psi(t)\rangle & =\sum_{j_{1}} \sum_{j_{2}} \ldots \sum_{j_{p}} A_{j_{1} j_{2} \ldots j_{p}}(t) \prod_{\kappa=1}^{p}\left|\varphi_{j_{\kappa}}^{(\kappa)}(t)\right\rangle, \\
\left|\varphi_{j_{\kappa}}^{(\kappa)}(t)\right\rangle & =\sum_{i_{1}} \sum_{i_{2}} \ldots \sum_{i_{Q(k)}} B_{i_{1} i_{2} \ldots i_{Q(\kappa)}}^{\kappa, j_{j}}(t) \prod_{q=1}^{Q(\kappa)}\left|v_{i_{q}}^{(\kappa, q)}(t)\right\rangle,
\end{aligned}
$$

$$
\left|v_{i_{q}}^{(\kappa, q)}(t)\right\rangle=\sum_{\alpha_{1}} \sum_{\alpha_{2}} \ldots \sum_{\alpha_{M(\kappa, q)}} C_{\alpha_{1} \alpha_{2} \ldots \alpha_{M(\kappa, q)}}^{\kappa, q, i_{q}}(t) \prod_{\gamma=1}^{M(\kappa, q)}\left|\xi_{\alpha_{\gamma}}^{\kappa, q, \gamma}(t)\right\rangle,
$$

where $A_{j_{1} j_{2} \ldots j_{p}}(t), B_{i_{1} i_{2} \ldots i_{Q(k)}}^{\kappa, j_{\kappa}}(t), C_{\alpha_{1} \alpha_{2} \ldots \alpha_{M(k, q)}, q, i_{q}}(t)$ etc. are the expansion coefficients for the first, second, third layers etc., respectively; $\left|\varphi_{j_{\kappa}}^{(\kappa)}(t)\right\rangle,\left|v_{i_{q}}^{(\kappa, q)}(t)\right\rangle,\left|\xi_{\alpha_{\gamma}}^{\kappa, q, \gamma}(t)\right\rangle$ etc. are the SPFs for the first, second, third layers etc., respectively. The notations beyond the first layer are as follows. In Eq. (2.22b) $Q(\kappa)$ is the number of (level 2) SP groups for the second layer that belong to the $\kappa$ th (level 1) SP group in the first layer, i.e., there are a total of $\sum_{\kappa=1}^{p} Q(\kappa)$ second layer SP groups. Continuing along the multilayer hierarchy, $M(\kappa, q)$ in Eq. (2.22) is the number of (level 3) SP groups for the third layer that belong to the $q$ th (level 2) SP group of the second layer and the $\kappa$ th (level 1) SP group of the first layer, resulting in a total of $\sum_{\kappa=1}^{p} \sum_{q=1}^{Q(\kappa)} M(\kappa, q)$ third layer SP groups. Such a recursive expansion can be carried out to an arbitrary number of layers. To terminate the multilayer hierarchy at a particular level, the SPFs in the deepest layer are expanded in terms of time-independent configurations. For example, in the four-layer version of the ML-MCTDH theory, the fourth layer is expanded in the time-independent basis functions/configurations, each of which may still contain several Cartesian degrees of freedom.

Applying the Dirac-Frenkel variational principle,

$$
\left\langle\delta \Psi(t)\left|i \frac{\partial}{\partial t}-\hat{H}\right| \Psi(t)\right\rangle=0
$$


with the functional form in Eq. (2.22), the equations of motion can be obtained as

$$
\begin{gathered}
i|\dot{\Psi}(t)\rangle_{\mathrm{L} 1 \text { coefficients }}=\hat{H}(t)|\Psi(t)\rangle, \\
i\left|\underline{\dot{\varphi}}^{k}(t)\right\rangle_{\mathrm{L} 2 \text { coefficients }}=\left[1-\hat{P}^{(\kappa)}(t)\right]\left[\hat{\rho}^{(\kappa)}(t)\right]^{-1}\langle\hat{H}\rangle^{(\kappa)}(t)\left|\underline{\varphi}^{(\kappa)}(t)\right\rangle,
\end{gathered}
$$

$$
\begin{aligned}
i\left|\underline{\dot{\underline{v}}}^{(\kappa, q)}(t)\right|_{\mathrm{L} 3 \text { coefficients }}= & {\left[1-\hat{P}_{\mathrm{L} 2}^{(\kappa, q)}(t)\right]\left[\hat{\varrho}_{\mathrm{L} 2}^{(\kappa, q)}(t)\right]^{-1} } \\
& \times\langle\hat{\mathcal{H}}\rangle_{\mathrm{L} 2}^{(\kappa, q)}(t)\left|\underline{v}^{(\kappa, q)}(t)\right\rangle, \quad(2 .
\end{aligned}
$$

$$
\begin{gathered}
i\left|\underline{\xi}^{(\kappa, q, \gamma)}(t)\right\rangle_{\mathrm{L} 4 \text { coefficients }}=\left[1-\hat{P}_{\mathrm{L} 3}^{(\kappa, q, \gamma)}(t)\right]\left[\hat{\varrho}_{\mathrm{L} 3}^{(\kappa, q, \gamma)}(t)\right]^{-1} \\
\times\langle\hat{\mathcal{H}}\rangle_{\mathrm{L} 3}^{(\kappa, q, \gamma)}(t)\left|\underline{\xi}^{(\kappa, q, \gamma)}(t)\right\rangle, \quad(2.2 \\
\ldots
\end{gathered}
$$

where the mean-field operators, reduced densities, and projection operators are defined in Ref. 7. For clarity we refer to the top layer as the level one (L1) SP space, the second layer as the level 2 (L2) SP space, etc. The time derivatives in Eq. (2.24), denoted by a overhead dot on the lefthand side of each equation, are meant to be carried out only with respect to the expansion coefficients of the particular layer which appeared in that equation. For example, the time derivative in Eq. (2.24a) acts only on the L1 expansion coefficient $A_{j_{1} j_{2} \ldots j_{p}}(t)$; the time derivative in Eq. (2.24b) is only on the L2 expansion coefficient $B_{i_{1} i_{2} \ldots i_{Q(k)}}^{\kappa, j_{k}}(t)$; etc. Note that for a $N$-layer version, there are $(N+1)$ levels of expansion coefficients because the SPFs in the deepest layer need to be expanded in time-independent basis functions/configurations. In this sense, the conventional wavepacket propagation method is a "zero-layer" MCTDH approach.

The inclusion of several dynamically optimized layers in the ML-MCTDH method provides more flexibility in the variational functional, which significantly advances the capabilities of performing wavepacket propagations in complex systems. This has been demonstrated by several applications to quantum dynamics in the condensed phase including many degrees of freedom. ${ }^{3,7,10,36-40}$ Further development is being pursued to incorporate more general forms of potential energy surfaces as well as to obtain a recursive implementation of the ML-MCTDH with an arbitrary number of layers. ${ }^{41,42}$ The method has also been implemented recently in the Heidelberg MCTDH package. ${ }^{43}$

The overall algorithm to evaluate the flux correlation function according to Eq. (2.19) can be summarized as follows:

- An initial state is sampled. To this end, a pseudorandom number generator is used to sample an initial bath state $|k\rangle$ according to the Boltzmann distribution $e^{-\beta E_{k}} / Q_{B}(T)$. For the model considered in this paper the "system" is one-dimensional such that there are only two eigenstates in Eq. (2.17). Therefore, instead of "sampling" the initial system state, we perform a direct summation: the sampled bath state $|k\rangle$ is simply combined with each of the two flux eigenstates $\left|u_{n=0,1}\right\rangle$ in turn.

- The initial state is then propagated in imaginary time according to Eq. (2.20). This is accomplished by setting $t=-i \beta$ in the ML-MCTDH equations of motion. ${ }^{3}$

- The resulting state $\left|\Phi_{n, k}^{\beta}\right\rangle$ is propagated in real time using the ML-MCTDH algorithm. The evolved state $e^{-i \hat{H} t}\left|\Phi_{n, k}^{\beta}\right\rangle$ is then projected onto the product region of coordinate space in order to construct the summand of Eq. (2.19).

This sequence is repeated for a number of samples sufficient to converge the summation in Eq. (2.19).

\section{MAPPING OF THE AZZOUZ-BORGIS MODEL TO A SYSTEM-BATH HAMILTONIAN}

The ML-MCTDH method is a general method for wavepacket propagation. However, it's rigorous and efficient application to systems with many degrees of freedom is currently limited to systems where the Hamiltonian can be written in the form of a sum of products of single-particle operators. The potential in the Azzouz-Borgis model is not of this form. To apply the ML-MCTDH method, we therefore employ a mapping of the original Hamiltonian of the Azzouz-Borgis model to a system-bath Hamiltonian that is tractable by the ML-MCTDH method.

\section{A. Mapping strategy}

This section describes an established protocol for mapping complex atomistic Hamiltonians onto simpler "systembath" models using classical molecular dynamics (MD) simulations. ${ }^{13,19-21}$ System-bath models describe the effect of the condensed-phase environment on the (small number of) coordinates of interest by coupling a "system" of low dimensionality to a large "bath" of harmonic oscillators. ${ }^{22,23}$ Since system-bath models are in the product form, the mapping procedure extends the applicability of the ML-MCTDH approach to condensed-phase reaction systems that are not described by the product-form Hamiltonians.

When there is just a single coordinate of interest, the system-bath Hamiltonian is

$$
H_{S B}=\frac{p_{s}^{2}}{2 m_{s}}+V_{s}(s)+\sum_{j}^{N}\left[\frac{P_{j}^{2}}{2 m_{j}}+\frac{1}{2} m_{j} \omega_{j}^{2}\left(Q_{j}-\frac{F_{j}(s)}{m_{j} \omega_{j}^{2}}\right)^{2}\right],
$$

where $s$ and $Q_{j}$ are position coordinates for the system and the $j^{\text {th }}$ bath mode respectively, and $p_{s}$ and $P_{j}$ are the conjugate momenta. The mass of the system is given by $m_{s}$, and $m_{j}$ denotes the mass of the $j^{\text {th }}$ bath mode. Since we are presently concerned with studying chemical reaction processes, the coordinate of interest $(s)$ will be a reaction coordinate, the potential energy along which is described by the function $V_{s}(s)$. In this work we assume bilinear system-bath coupling, such that $F_{j}(s)=c_{j} s$. The coupling coefficients $\left(\left\{c_{j}\right\}\right)$ and bath 
frequencies $\left(\left\{\omega_{j}\right\}\right)$ are combined in the spectral density

$$
J(\omega)=\frac{\pi}{2} \sum_{j=1}^{N} \frac{c_{j}^{2}}{m_{j} \omega_{j}} \delta\left(\omega-\omega_{j}\right),
$$

which comprises all information of the bath that influences the dynamics of the reaction coordinate $s$.

Having briefly introduced the system-bath model, now consider the case where we have some complicated atomistic Hamiltonian providing a reasonably realistic description of a chemical reaction of interest, but which is not in product form. In order to map this Hamiltonian onto the system-bath model (thus enabling the application of the ML-MCTDH approach) two issues must be resolved. The first of these is the precise identity of the potential energy function $V_{s}(s)$. This is revealed by considering that within the system-bath model the force on $s$,

$$
F_{S}(s)=-\frac{\partial H_{S B}}{\partial s}=-\frac{d V_{s}(s)}{d s}+\sum_{j}^{N}\left[c_{j} Q_{j}-\frac{c_{j}^{2} s}{m_{j} \omega_{j}^{2}}\right],
$$

when averaged over the bath modes (at some fixed $s=s^{*}$ ), is:

$$
\left\langle F_{s}\left(s^{*}\right)\right\rangle \equiv \frac{\operatorname{tr}\left[e^{-\beta H_{S B}} \delta\left(s-s^{*}\right) F_{s}(s)\right]}{\operatorname{tr}\left[e^{-\beta H_{S B}} \delta\left(s-s^{*}\right)\right]}=-\left.\frac{d V_{s}(s)}{d s}\right|_{s=s^{*}} .
$$

This shows that $V_{s}(s)$ is the potential of mean force along the reaction coordinate, ${ }^{28}$ and, furthermore, demonstrates that the bath modes do not exert an average (static equilibrium) force on the reaction coordinate. Instead, their only effect is to cause friction for the reaction coordinate.

The second issue to be resolved is the identity of the spectral density function Eq. (3.2), which defines the distribution of bath frequencies and coupling coefficients. The spectral density is related via ${ }^{44}$

$$
J(\omega)=\omega \int_{0}^{\infty} d t \eta(t) \cos (\omega t),
$$

with

$$
\eta(t)=\beta\left\langle F_{s}(0) F_{s}(t)\right\rangle,
$$

to the force-force autocorrelation function $\left\langle F_{s}(0) F_{s}(t)\right\rangle$, which is evaluated with the reaction coordinate $s$ constrained at a fixed value. The spectral density is therefore a thermallyweighted frequency-domain representation of the fluctuations in the force on $s$.

In practical terms then, the mapping involves the calculation of both the potential of mean force along the reaction coordinate $\left[V_{s}(s)\right]$ and the force-force autocorrelation function $\left[\left\langle F_{s}(0) F_{s}(t)\right\rangle\right]$. For an atomistic Hamiltonian both tasks are conveniently accomplished with classical molecular dynamics (MD) calculations. ${ }^{45}$ For example, by adding appropriate constraint forces ${ }^{46}$ to the dynamics of the atomistic Hamiltonian, the mean force on the reaction coordinate [Eq. (3.4)] can be determined at a set of fixed grid points along $s$. Integration then yields the potential of mean force

$$
V_{s}(s)=V_{s}\left(s_{0}\right)+\int_{s_{0}}^{s} d s_{1}\left\langle F_{s}\left(s_{1}\right)\right\rangle .
$$

Similarly, $\eta(t)$ can be obtained from an appropriately constrained MD simulation and yields $J(\omega)$ after performing the cosine transform in Eq. (3.5).

A well-established and efficient procedure can be used to obtain a set of bath frequencies and coupling constants from this spectral density. ${ }^{47}$ In brief, this involves determining the bath frequencies $\left(\left\{\omega_{j}\right\}\right)$ by locating the zero value of the function

$$
f\left(\omega_{j}\right)=\left(\int_{0}^{\omega_{j}} d \omega \rho(\omega)\right)-j
$$

for $j=1, \ldots, N$, where $\rho(\omega)=J(\omega) / \omega$. Having selected the bath frequencies, the coupling coefficients are then given by ${ }^{47}$

$$
c_{j}^{2}=\omega_{j} \frac{2 m_{j}}{\pi} \frac{J\left(\omega_{j}\right)}{\rho\left(\omega_{j}\right)} .
$$

The mapping strategy based on classical MD simulations has been applied in previous work of several groups to extract spectral densities from complicated atomistic Hamiltonians and thus map them onto more tractable system-bath models, see, e.g., Refs. 13, 19-21, 48-50. It should be noted that the mapping involves a number of implicit assumptions:

- The reactive process can be accurately characterized as motion along the single system (i.e., reaction) coordinate, and that the environment acts on this coordinate as a large set of independent degrees of freedom corresponding to the linear response regime. Makri has discussed the conditions which are necessary for the validity of this assumption. ${ }^{44}$

- The effect of the environment on the reaction coordinate is independent of the position along that reaction coordinate, i.e., that $J(\omega)$ does not depend on which fixed value of $s$ is employed in the MD calculation of $\left\langle F_{s}(0) F_{s}(t)\right\rangle$. In order to accurately model any $s$-dependency of $J(\omega)$ the system-bath coupling $\left(F_{j}(s)\right)$ would have to be a nonlinear function in $s .{ }^{51,52}$

- The dynamics of the reaction coordinate are governed by the potential of mean force. This requires the dynamical timescales of the environmental modes to be sufficiently short such that there is an adiabatic separation from the reactive motion.

- The potential of mean force and spectral density obtained from classical MD simulations are reasonable approximations to their quantum-mechanical counterparts. ${ }^{13}$

The extent to which these assumptions hold for a particular system is not always obvious in advance. However, later sections present an a posteriori analysis of the reliability of the mapping strategy for the specific case of the Azzouz-Borgis model considered in this work. 


\section{B. The Azzouz-Borgis model}

The Azzouz-Borgis model describes a proton transfer reaction occurring in a polar aprotic solvent. ${ }^{11}$ Since it accounts for many of the important features of such reactions at a pseudo-atomistic level of description, it has been the target of a number of studies employing various approximate quantum-mechanical and quantum-classical methods for calculating thermal rate constants. ${ }^{12-18}$ The results of these studies span quite a wide range of values - in part due to reasons that are not well understood. ${ }^{18}$ Employing the mapping of the original Azzouz-Borgis model to a system-bath Hamiltonian the ML-MCTDH method can provide accurate benchmark results of the proton-transfer rate to validate the approximate methods. In this section, we use the strategy described above to map the Hamiltonian $H_{A B}$ onto a system-bath model.

The specific reaction process considered by the AzzouzBorgis model is the proton transfer between phenol (proton donor, A) and trimethylamine (proton acceptor, B) in liquid methyl chloride. ${ }^{11}$ The potential energy function of the model is

$$
\begin{aligned}
V_{A B}\left(r, \mathbf{r}_{A}, \mathbf{r}_{B}, \mathbf{r}_{S}\right)= & V_{C}\left(r, \mathbf{r}_{A}, \mathbf{r}_{B}\right)+V_{S}\left(\mathbf{r}_{S}\right) \\
& +V_{C S}\left(r, \mathbf{r}_{A}, \mathbf{r}_{B}, \mathbf{r}_{S}\right),
\end{aligned}
$$

where $V_{C}$ is the potential for the donor-proton-acceptor (AHB) complex, $V_{S}$ is that of the solvent, and $V_{C S}$ describes the interaction between the complex and the solvent. The scalar coordinate $r$ is the distance of the proton from A along the A-B axis, whereas $\mathbf{r}_{A}, \mathbf{r}_{B}$, and $\mathbf{r}_{S}$ are respectively the Cartesian position vectors of $\mathrm{A}, \mathrm{B}$, and the solvent sites. One simplifying feature of the model is that the A (phenol) and B (trimethylamine) molecules are each represented with a single spherical particle of appropriate mass, whilst each solvent molecule is comprised of one methyl site and one chloride site at fixed separation. A second simplification is that the AHB complex is constrained to be collinear.

The potential of the AHB complex is a function of both $r$ and the donor-acceptor distance $R=\left|\mathbf{r}_{A}-\mathbf{r}_{B}\right|$,

$$
\begin{aligned}
V_{C}(r, R)= & b e^{-a R}+D_{A}\left[1-e^{-n_{A}\left(r-d_{A}\right)^{2} / 2 r}\right] \\
& +D_{B}\left[1-e^{-n_{B}\left(R-r-d_{B}\right)^{2} / 2(R-r)}\right] .
\end{aligned}
$$

The values of the parameters in this equation, and of all other parameters in the model, were set equal to those used by Hammes-Schiffer and Tully. ${ }^{12,16}$

In the solvent, each pair of methyl chloride molecules interacts via a potential which is a sum of Coulombic and Lennard-Jones terms. Likewise, each solvent site (whether methyl or chloride) interacts with the A and B sites of the donor-proton-acceptor complex via Lennard-Jones terms, and with $\mathrm{A}, \mathrm{B}$, and the proton via Coulombic terms. The electrostatic interactions involving A and B clearly depend on the partial charges of these sites, which vary with the position of the proton. ${ }^{11}$ Thus, as the proton transfer proceeds, the AHB complex develops a large dipole moment that is strongly solvated by the polar solvent. This solvation stabilizes the ionic $\mathrm{A}^{-}-\mathrm{HB}^{+}$product with respect to the neutral $\mathrm{AH}-\mathrm{B}$ reactant, which is the stable species in the gas-phase. The solvent is therefore a fundamental component in this reaction process.
More detailed descriptions of the Azzouz-Borgis Hamiltonian can be found in the literature. ${ }^{11,12,16}$

We now turn to consider the mapping of $H_{A B}$ onto a system-bath model. As discussed in the previous section, a principal step in this process is the selection of a "system" (i.e., reaction) coordinate, $s$, from among the many degrees of freedom involved in the original Hamiltonian. Here we have used the Azzouz-Borgis proton transfer distance, $r$. In particular, we choose

$$
s=r-r_{s},
$$

where $r_{s}$ is a shift discussed below. Having made this choice, the two key components of the mapped system-bath model must be determined, i.e., the potential of mean force along the reaction coordinate $\left(V_{s}(s)\right)$ and the force-force autocorrelation function $\left(\left\langle F_{s}(0) F_{s}(t)\right\rangle\right)$. Due to Eq. (3.12), the former is simply related to $\bar{V}(r)$, the potential of mean force along the proton transfer coordinate of the original Azzouz-Borgis model. In order to construct this $\bar{V}(r)$, the mean force

$$
\left\langle F_{r}\left(r^{*}\right)\right\rangle=\frac{\operatorname{tr}\left[e^{-\beta H_{A B}} \delta\left(r-r^{*}\right)\left(-\frac{\partial V_{A B}}{\partial r}\right)\right]}{\operatorname{tr}\left[e^{-\beta H_{A B}} \delta\left(r-r^{*}\right)\right]},
$$

was first evaluated for a grid of points $\left(r^{*}\right)$ along $r$ using classical MD simulations in which the proton-transfer coordinate $r$ was held fixed with standard Lagrangian constraint methods. ${ }^{46}$ Following previous work, our simulations included 255 solvent molecules at a temperature of $\mathrm{T}=249 \mathrm{~K}$ and a density of $0.0117 \AA^{-3}$. The minimum image convention was used to implement periodic boundary conditions, and a Steinhauser function ${ }^{12}$ was applied to truncate the Coulombic interactions at a distance of $13.8 \AA$. At each grid point, the mean force $\left\langle F_{r}\left(r^{*}\right)\right\rangle$ was determined from 1000 trajectories of length $5 \mathrm{ps}$ and timestep 5 fs. Each trajectory started from the final position of the previous one, but the initial momenta were re-sampled from the Boltzmann distribution in order produce results consistent with the canonical ensemble. After integrating $\left\langle F_{r}\left(r^{*}\right)\right\rangle$ to yield $\bar{V}(r)$ (in the manner of Eq. (3.7)), we have

$$
V_{s}(s)=\bar{V}\left(s+r_{s}\right) .
$$

The value of the shift $r_{s}=1.30 \AA$ is determined such that $s$ $=0$ coincides with the position of the barrier in $V_{s}(s)$.

An interesting point is that the potential energy function of the AHB complex [Eq. (3.11)] is dissociative along $R$. Consequently, it can be shown that the mean force on $r$ [Eq. (3.13)] is determined by the force on $r$ in the asymptotic $(R \rightarrow \infty)$ limit. In order to maintain a focus on the protontransfer region of the potential, we have therefore augmented $V_{C}(r, R)$ with a term which is attractive along the donoracceptor coordinate $R$ and which prevents dissociation:

$$
V_{C}(r, R) \longrightarrow V_{C}(r, R)+b e^{+a\left(R-R_{b}\right)} .
$$

Here, the parameters $a$ and $b$ are identical to those in the similar (but repulsive) leading term of Eq. (3.11), and $R_{b}=6.0 \AA$. The new term is negligible in the small- $R$ interaction region $(R \sim 2.7 \AA)$, e.g., the change in the energy barrier is less than $0.005 \mathrm{kcal} \mathrm{mol}^{-1} .^{53}$

The potential of mean force that results from the MD simulation is plotted as a function of the system-bath 


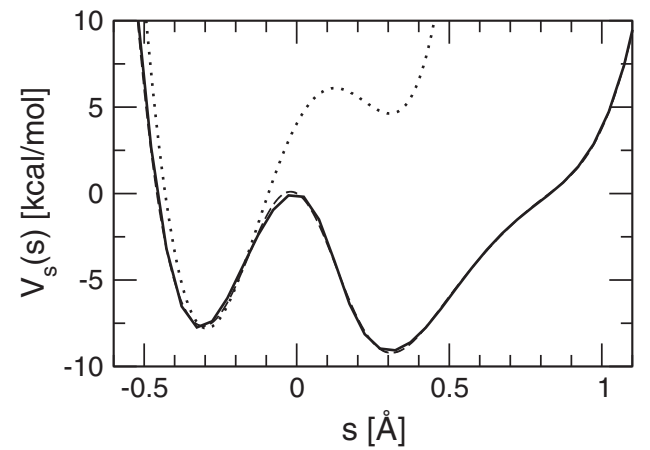

FIG. 1. Potential of mean force along the system/reaction coordinate of the system-bath model in Eq. (3.1) (solid line). Also plotted is the polynomial fit to the numerical data [Eq. (4.9); dashed line] and a cut at $R$ $=2.7 \AA$ through the gas-phase potential of the donor-proton-acceptor complex $V_{C}(r, R)$ [Eq. (3.11); dotted line].

reaction coordinate $s$ in Fig 1. For comparison, a cut through the two-dimensional potential of the AHB complex (the augmented $V_{C}(r, R)$ ) along $r$ at constant $R=2.7 \AA$ is also plotted (as a function of $s=r-r_{s}$ ). The previously-mentioned stabilization of the ionic product by the polar solvent is clearly apparent at $s>0$. Following the arguments presented in Sec. III, this large static-equilibrium effect would be absent from the mapped model were we to simply set $V_{s}(s)$ equal to a cut through the (gas-phase) AHB potential, $V_{C}(r, R) .{ }^{13} \mathrm{It}$ should be noted, though, that the use of the potential of mean force for the dynamics of the proton, in particular for short timescales, has to be considered with caution. The low characteristic frequency of the spectral density (see below) suggests that the proton is subject to forces derived from instantaneous bath configurations. These concerns are addressed in Sec. IV by comparing the classical reactive dynamics and rate constants of the system-bath model with those of the original Azzouz-Borgis Hamiltonian. A second set of MD simulations was used to evaluate the classical force-force autocorrelation function $\left(\left\langle F_{S}(0) F_{S}(t)\right\rangle\right)$ and thus determine the spectral density $(J(\omega))$ via Eqs. (3.5) and (3.6). In order to accurately describe the effect of the environment on the proton in the crucial barrier-crossing region, we followed previous work and evaluated the force correlation function with the proton constrained near the barrier top $(r=1.34 \AA) .{ }^{13,54,55}$ The remaining computational details were identical to those used to calculate $\left\langle F_{r}\left(r^{*}\right)\right\rangle$, except that $10^{5}$ trajectories of length $10 \mathrm{ps}$ and a timestep of 1 fs were used to construct $\left\langle F_{r}(0) F_{r}(t)\right\rangle$ and thus $\left\langle F_{s}(0) F_{s}(t)\right\rangle$. The resulting spectral density, shown in Fig. 2, has two components. The broad band at low frequency arises from forces exerted on the proton by the methyl chloride solvent. It has a maximum at $\omega=42 \mathrm{~cm}^{-1}$, corresponding to a period of about $800 \mathrm{fs}$. The second feature in $J(\omega)$ is the sharp spike around $\sim 690 \mathrm{~cm}^{-1}$. This can be assigned to the relative $\mathrm{A}-\mathrm{B}$ motion (the "donor-acceptor" mode) since the harmonic frequency of $V_{C}(r, R)$ (Eq. (3.11)) along $R$ at $r=1.34 \AA$ is almost exactly $690 \mathrm{~cm}^{-1}$. ${ }^{56}$ The finite width of this peak is presumably due to the anharmonicity of potential and/or broadening by the solvent.

The spectral density associated with the proton-transfer coordinate of the Azzouz-Borgis model has been previ-

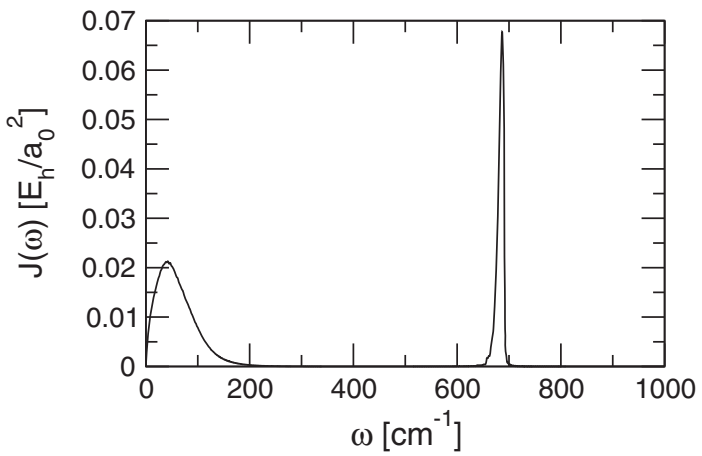

FIG. 2. Spectral density of the Azzouz-Borgis model calculated at $r$ $=1.34 \AA$ as described in Sec. III.

ously calculated by Antoniou and Schwartz (see Fig. 2 of Ref. 13). Our result differs from the earlier one in a few respects. Firstly, the previous result has no high-frequency component. ${ }^{13}$ Secondly, the position of the maximum in the earlier result disagrees with that of the low-frequency band seen in Fig. 2. Thirdly, the previously-calculated spectral density has a shoulder on the high-frequency side of the (lowfrequency) maximum which is absent from the present result.

Based on the available information in Ref. 13, it is difficult to trace the the origin of the discrepancies. It seems to us that one should expect a high-frequency component in $J(\omega)$ since the high-frequency donor-acceptor mode $(R)$ is strongly coupled to the proton-transfer coordinate $(r)$ by the potential $V_{C}(r, R)$. The discrepancies might also be due to the use of different constrained values of $r$. The authors of the previous work have already established that the spectral density may depend on the constrained value of the proton-transfer coordinate, which is used to calculated the force-force autocorrelation function. ${ }^{57}$

\section{RESULTS}

The methodology outlined above has been applied to study the proton transfer dynamics of the Azzouz-Borgis model, in particular to calculate the thermal rate constant. In this section we present the results of the rate calculations and analyze the underlying dynamics. Furthermore, a comparison of the classical dynamics of the original Azzouz-Borgis model with that of the mapped linear response model illustrates the mapping procedure.

\section{A. Classical dynamics}

We first consider the classical dynamics of the AzzouzBorgis model. In contrast to the quantum dynamics, the classical dynamics of the original Azzouz-Borgis model can be obtained rather straightforwardly. The exact classical flux correlation functions $\left(C_{f}^{\mathrm{cl}}(t)\right)$ of the original and the mapped version of the model can be calculated, e.g., using the BennettChandler method. ${ }^{27,46,58}$ By comparing the two results one can then establish whether or not the system-bath mapping provides a reasonable approximation to the reactive dynamics and rate constants of the original model. This approach therefore provides valuable information on the validity of the 
assumptions that are implicit in the mapping procedure described above (see Sec. III A).

For a reactive system with $n$ degrees of freedom, the classical flux correlation function is

$$
\begin{aligned}
C_{f}^{\mathrm{cl}}(t)= & \frac{1}{(2 \pi \hbar)^{n}} \int d \mathbf{p}_{0} \int d \mathbf{q}_{0} e^{-\beta H\left(\mathbf{p}_{0}, \mathbf{q}_{0}\right)} \delta\left[s\left(\mathbf{q}_{0}\right)\right] \\
& \times v_{s}\left(\mathbf{p}_{0}, \mathbf{q}_{0}\right) h\left[s\left(\mathbf{q}_{t}\right)\right]
\end{aligned}
$$

where the position coordinates are $\mathbf{q}=\left(s,\left\{Q_{j}\right\}\right)$ for the system-bath model and $\mathbf{q}=\left(r, \mathbf{r}_{A}, \mathbf{r}_{B}, \mathbf{r}_{S}\right)$ for the original Azzouz-Borgis model. In each case $\mathbf{p}$ denotes the momenta conjugate to $\mathbf{q}, \delta\left[s\left(\mathbf{q}_{0}\right)\right]$ constrains the initial value of the reaction coordinate to the dividing surface, $v_{s}\left(\mathbf{p}_{0}, \mathbf{q}_{0}\right)$ is the initial velocity along the reaction coordinate, and $h\left[s\left(\mathbf{q}_{t}\right)\right]$ is the product side-function evaluated at time $t$. Rather than $C_{f}^{\mathrm{cl}}(t)$ itself, here we consider the ratio $C_{f}^{\mathrm{cl}}(t) / Q_{r}^{\mathrm{cl}}(T)$ (where $Q_{r}^{\mathrm{cl}}(T)$ is the classical reactant partition function) since this quantity determines the classical rate constant under the conditions in which Eq. (2.11) is valid. The ratio can be expressed as a product of the transition-state theory (TST) rate constant and a time-dependent transmission coefficient ${ }^{46}$

$$
C_{f}^{\mathrm{cl}}(t) / Q_{r}^{\mathrm{cl}}(T)=k_{\mathrm{TST}}^{\mathrm{cl}}(T) \kappa^{\mathrm{cl}}(t),
$$

where

$$
\begin{aligned}
\kappa^{\mathrm{cl}}(t)= & \frac{C_{f}^{\mathrm{cl}}(t)}{(2 \pi \hbar)^{n}} \int d \mathbf{p}_{0} \int d \mathbf{q}_{0} e^{-\beta H\left(\mathbf{p}_{0}, \mathbf{q}_{0}\right)} \delta\left[s\left(\mathbf{q}_{0}\right)\right] \\
& \times v_{s}\left(\mathbf{p}_{0}, \mathbf{q}_{0}\right) h\left[p_{s}\left(\mathbf{p}_{0}, \mathbf{q}_{0}\right)\right],
\end{aligned}
$$

and $h\left[p_{s}\left(\mathbf{p}_{0}, \mathbf{q}_{0}\right)\right]$ is unity if the initial momentum along the reaction coordinate is positive and is zero otherwise.

Both the numerator and the denominator of the transmission coefficient in Eq. (4.3) for the original Azzouz-Borgis model can be evaluated by first sampling position coordinates located at the dividing surface between reactants and products. In order that this sampling is consistent with the Boltzmann distribution, one can randomly select configurations from a constant-temperature molecular dynamics simulation in which the reaction coordinate is constrained to the dividing surface. An initial phase-space point for a classical trajectory is then produced by sampling a set of momenta according to $e^{-(\beta / 2) \mathbf{p}_{0} \cdot \mathbf{M}^{-1} \cdot \mathbf{p}_{0}}$, where $\mathbf{M}$ is the mass matrix. For each point sampled in this way, the constraint on the reaction coordinate is released, and the path of the resulting trajectory is followed in order to evaluate its contribution to the ensemble averages in the numerator and denominator of Eq. (4.3).

In our calculation, the constrained dividing-surface MD simulation ran for $25 \mathrm{ps}$ with a time step $0.25 \mathrm{fs}$. An Andersen thermostat ${ }^{59}$ was used to control the temperature by resampling the momenta around once in every 50 time steps. At intervals of exactly 400 time steps (100 fs) along this constrained dividing-surface trajectory, two unconstrained trajectories were initiated as described above and their motion away from the dividing surface was followed for $4 \mathrm{ps}$, again with a time step of $0.25 \mathrm{fs}$. Each constrained dividing-surface MD run thus produced five hundred unconstrained trajectories. Repeating the dividing-surface MD with forty different values of the initial random number generator seed, we ob- tained a well-converged result for $\kappa^{\mathrm{cl}}(t)$ from twenty thousand unconstrained trajectories.

According to Eq. (4.2), in order to translate $\kappa^{\mathrm{cl}}(t)$ into the time-dependent ratio $C_{f}^{\mathrm{cl}}(t) / Q_{r}^{\mathrm{cl}}(T)$, and thus the classical rate constant, the classical transition-state theory rate constant,

$$
k_{\mathrm{TST}}^{\mathrm{cl}}(T)=\frac{1}{\sqrt{2 \pi m \beta}} p\left(r_{\ddagger}\right),
$$

is required, Thereby,

$$
p(r)=p\left(r_{0}\right) e^{-\beta\left(\bar{V}(r)-\bar{V}\left(r_{0}\right)\right)},
$$

denotes the probability distribution for the proton transfer coordinate, $r_{\ddagger}=1.30 \AA$ is the chosen dividing surface, and $\bar{V}(r)$ is the potential of mean force that was calculated in Sec. III. Thus, the only additional information needed to compute $p\left(r_{\ddagger}\right)$ (and thus $k_{\mathrm{TST}}^{\mathrm{cl}}(T)$ ) is $p\left(r_{0}\right)$, the probability distribution at some other arbitrary $r=r_{0}$. A convenient choice places $r_{0}$ near the minimum of the reactant well, since $p\left(r_{0}\right)$ can then be efficiently determined from a standard unconstrained MD simulation. ${ }^{18}$ We used $r_{0}=1.008 \AA$ and found that $10^{4}$ trajectories of length $10 \mathrm{ps}$ and time step $1 \mathrm{fs}$ were sufficient to give a converged result of $p\left(r_{0}\right)=13.15 \AA^{-1}$. According to Eqs. (4.4) and (4.5), the classical transition-state theory rate constant of the original model is therefore $k_{\mathrm{TST}}^{\mathrm{cl}}(T)$ $=1.43 \times 10^{-3} 10^{10} \mathrm{~s}^{-1}$.

The TST rate constant can alternatively be obtained from $^{18,46}$

$$
k_{\mathrm{TST}}^{\mathrm{cl}}(T)=\frac{1}{\sqrt{2 \pi m \beta}} \frac{e^{-\beta \bar{V}\left(r_{\ddagger}\right)}}{\int_{0}^{r_{\ddagger}} d r e^{-\beta \bar{V}(r)}} .
$$

This equation implies that the mapped model should give exactly the same transition-state theory rate constant as the original Azzouz-Borgis Hamiltonian, since the potential of mean force in the system-bath model $\left(V_{s}(s)\right)$ is constructed to equal $\bar{V}(r)$ (a consequence of Eq. (3.12)). However, $k_{\mathrm{TST}}^{\mathrm{cl}}(T)$ is sensitive to numerical errors in $\bar{V}(r)$, since it appears in the exponential in Eq. (4.6). The difference of the TST rate constant obtained from Eq. (4.4) and that calculated by inserting the system-bath potential of mean force into Eq. (4.6) thus gives an estimate of the numerical error in the calculation (see Table I).

A different method was used to calculate $C_{f}^{\mathrm{cl}}(t) / Q_{r}^{\mathrm{cl}}(T)$ for the system-bath mapping of the Azzouz-Borgis model. Specifically, the flux correlation function $C_{f}^{\mathrm{cl}}(t)$ was evaluated using the classical analog of the dividing-surface sampling approach discussed in Sec. V of Ref. 60. In brief, this approach draws initial phase-space points for classical trajectories from a normalized Gaussian sampling function which is defined in terms of a set of normal-mode coordinates. For the calculation

TABLE I. Classical rate constants for the Azzouz-Borgis model.

\begin{tabular}{lcc}
\hline \hline & Original model & System-bath model \\
\hline $\mathrm{k}_{\mathrm{TST}}^{\mathrm{cl}}\left[10^{10} \mathrm{~s}^{-1}\right]$ & $1.43 \times 10^{-3}$ & $1.32 \times 10^{-3}$ \\
$\kappa(t \rightarrow \infty)[$ dimensionless $]$ & 0.133 & 0.0877 \\
$\mathrm{k}_{\mathrm{CL}}\left[10^{10} \mathrm{~s}^{-1}\right]$ & $1.90 \times 10^{-4}$ & $1.16 \times 10^{-4}$ \\
\hline
\end{tabular}


of the flux correlation function, the normal modes are evaluated at the dividing surface of the system-bath model. After this sampling, the trajectories are propagated in time in order to evaluate the time-dependent terms in the integrand of $C_{f}^{\mathrm{cl}}(t)$. At T $=249 \mathrm{~K}$, a tightly converged $C_{f}^{\mathrm{cl}}(t)$ was obtained using $10^{6}$ trajectories with a timestep of $\sim 1$ fs. For more details on the dividing-surface sampling approach see Ref. 60. Because the potential is harmonic along the bath mode coordinates, the classical reactant partition function for the systembath mapping can be written as

$$
Q_{r}^{\mathrm{cl}}(T)=\chi(T) \int_{-\infty}^{0} d s^{*} e^{-\beta V_{s}\left(s^{*}\right)},
$$

and

$$
\chi(T)=\sqrt{\frac{m}{2 \pi \hbar^{2} \beta}} \prod_{j=1}^{n-1} \beta \hbar \omega_{j},
$$

where $\left\{\omega_{j}\right\}$ are the bath frequencies discussed in the context of Eq. (3.1). The classical reactant partition function of the system-bath model can therefore be calculated by integrating $e^{-\beta V_{s}\left(s^{*}\right)}$ along the reaction coordinate $s$.

In the interests of brevity, no further methodological details of the classical calculations are given here except for the following two important points. Firstly, to enable the propagation of classical trajectories on the system-bath potential energy surface, the numerical data for the potential of mean force $\left(V_{s}(s)\right)$ were fitted to simple polynomial expansion,

$$
V_{s}(s)=\sum_{j=0}^{M} a_{j} s^{j},
$$

using a standard linear least-squares fitting procedure. ${ }^{61} \mathrm{We}$ took $M=18$, giving a root mean-square deviation from the original data of $0.14 \mathrm{kcal} \mathrm{mol}^{-1}$ and a maximum deviation of $0.35 \mathrm{kcal} \mathrm{mol}^{-1}$. The polynomial fit is plotted alongside the numerical $V_{s}(s)$ in Fig. 1. Secondly, Eqs. (3.8) and (3.9) must be used to discretize the spectral density so that it can be represented with a finite number of bath modes [see Eq. (3.2)]. In order to perform the integration in Eq. (3.8) we used a spline interpolation $^{61}$ of the numerical data for the density of states $\rho(\omega)$.

The time-dependent ratio $C_{f}^{\mathrm{cl}}(t) / Q_{r}^{\mathrm{cl}}(T)$ is presented for both the system-bath mapping and the original Azzouz-Borgis model in Fig. 3. As $t \rightarrow 0$ these results give the classical transition-state theory rate constant for their respective models since $\kappa(t \rightarrow 0)=1$. In addition, as $\mathrm{t} \rightarrow \infty$ this ratio $C_{f}^{\mathrm{cl}}(t) / Q_{r}^{\mathrm{cl}}(T)$ determines the full classical rate constant according to the appropriate equation from Sec. II. The full classical and classical transition-state theory rate constants corresponding to Fig. 3 are presented in Table 1 for both models. The full rate constant for the original Azzouz-Borgis model has been calculated using Eq. (2.10) since $P_{r}(0)=1$ classically. Despite the use of Eq. (2.10), which gives a more rigorous description of the rate constant, the results in Fig. 3 show that the classical flux correlation function of the original Azzouz-Borgis model does not reach a strict plateau but exhibits even for longer times (data not shown) a small but noticeable slope. This indicates that the proton transfer reaction

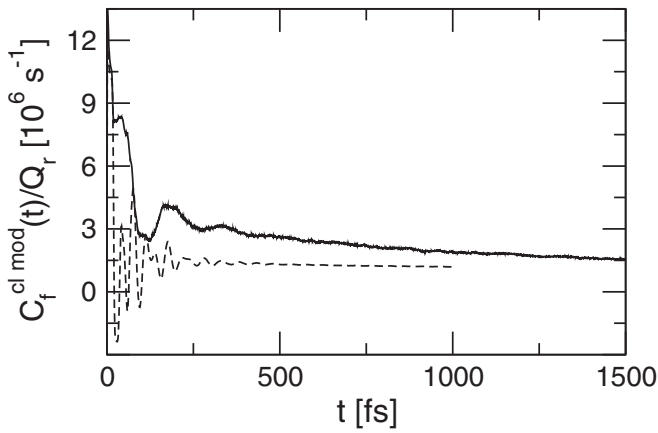

FIG. 3. Classical flux correlation functions for the Azzouz-Borgis proton transfer system. Solid line: original model. Dotted line: mapped system-bath model. Shown is the modified classical flux correlation function as calculated by Eq. (2.10), i.e., $C_{f}^{\mathrm{cl} \bmod }(t)=C_{f}^{\mathrm{cl}}(t) /\left[1-\left(Q_{r}(T)^{-1}\right.\right.$ $\left.\left.+Q_{p}(T)^{-1}\right) \int_{0}^{t} d \tau C_{f}(\tau)\right]$. For the system-bath model, $C_{f}^{\text {cl mod }}(t)$ agrees with the commonly used form $C_{f}^{\mathrm{cl}}(t)$. In each case, the short-time limit gives the classical transition-state theory rate constant and the long-time limit gives the full thermal rate constant.

in the original Azzouz-Borgis model is not a true rate process and strictly-speaking cannot be described by a (single) rate constant. Since the slope of the flux correlation function is, however, rather small, an approximate rate constant can be defined. Here, we have used the value at $t=1 \mathrm{ps}$ to estimate the rate constant. For the system-bath model, on the other hand, the plateau observed in Fig. 3 indicates a sufficient separation of reactive and nonreactive timescales such that Eq. (2.11) is valid.

Although there are differences between the two results depicted in Fig. 3, in particular in the transient short time dynamics, it is apparent that for longer times, $t>250 \mathrm{fs}$, the reactive dynamics of the system-bath model are similar to those of the original Azzouz-Borgis Hamiltonian. This agreement, also seen in the numerical results in Table I, is remarkable considering the approximations required to derive the systembath mapping in Sec. III A and suggests that the mapped model is a useful approximation to the original AzzouzBorgis system provided that one is interested in calculating $k(T)$. The discrepancies observable in the system-bath result, such as the rapid short-time oscillations at $t<250 \mathrm{ps,} \mathrm{are} \mathrm{a}$ consequence of the oversimplicity of the model. For example, the model assumes position-independent friction, while the friction exerted on the reaction coordinate at the reactant state might be different to that exerted at the transition state. Possible extensions of the system-bath model to improve the representation of the original Azzouz-Borgis model are discussed in Sec. V.

Although the system-bath model shows significant deviations for the short-time transient dynamics, the results in Fig. 3 imply that the assumptions involved in deriving the system-bath model are justified for the description of the longtime dynamics and the thermal rate constant, which is the main objective of this paper. In Sec. IV B the ML-MCTDH approach will be applied to the system-bath model in order to perform an accurate quantum-mechanical evaluation of the proton-transfer rate constant for the Azzouz-Borgis system. 


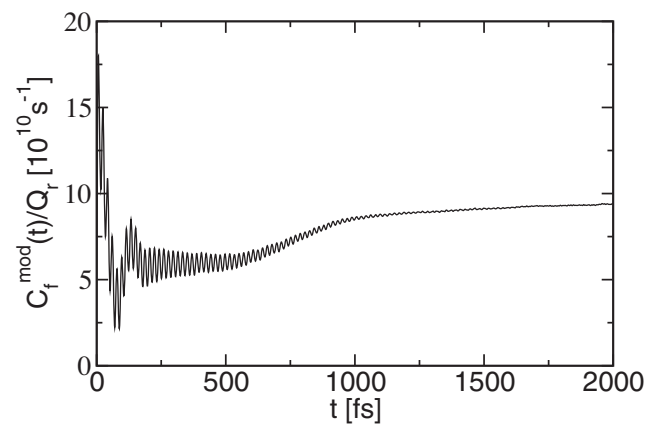

FIG. 4. Quantum flux correlation function for the system-bath mapping of the Azzouz-Borgis model, as calculated by the ML-MCTDH approach described in Sec. II. Shown is the modified flux correlation function as calculated by Eq. (2.9), i.e., $C_{f}^{\bmod }(t)=C_{f}(t) /\left[P_{r}(0)+\left(P_{r}(0)\right.\right.$ $\left.-1)\left(Q_{r}(T) / Q_{p}(T)\right)\right]$.

\section{B. Quantum dynamics}

The quantum-mechanical flux correlation function for the system-bath mapping of the Azzouz-Borgis Hamiltonian is presented in Fig. 4, normalized by the quantum-mechanical reactant partition function. The numerically-converged result was obtained using the ML-MCTDH approach discussed in Sec. II, and the parameters given in Table II.

A number of features are worthy of comment. Firstly, the overall amplitude of the correlation function is about four orders of magnitude larger than either of the classical correlation functions in Fig. 3. This represents a large quantummechanical enhancement of the proton-transfer reaction rate for this system. Secondly, the details of the quantum reactive dynamics of the system-bath model are rather different from the classical counterpart shown above. For example, although both classical and quantum correlation function show oscillatory behavior, the oscillations in the quantum results have higher frequency and are more regular. It should be stressed that these oscillations are not artifacts of insufficient convergence. A third difference between Figs. 3 and 4 is that the extent of the dividing-surface recrossing, i.e., the difference between the short- and long-time limits of $C_{f}(t)$, is greatly reduced in the quantum-mechanical result.

The observation that the quantum-mechanical flux correlation function reaches a time-independent plateau at $t$ $>1.5$ ps implies that Eq. (2.9) is appropriate for calculating the full quantum-mechanical rate constant. The denominator on the right-hand-side of Eq. (2.9) was again evaluated via the ML-MCTDH simulation according to the

TABLE II. Values of some important ML-MCTDH convergence parameters.

\begin{tabular}{lcc}
\hline \hline & $C_{f}(t)$ & $Q_{r}(T)$ \\
\hline Number of bath modes & 100 & 100 \\
Number of sampled initial states & 500 & 20000 \\
Number of layers & 4 & 4 \\
Number of first-layer SP groups & 4 & 4 \\
Number of first-layer SP functions & 16 & 16 \\
\hline \hline
\end{tabular}

TABLE III. Results of previous approximate quantum-mechanical calculations of the proton-transfer rate constant of the Azzouz-Borgis model. Adapted from Refs. 18. Units are $10^{10} \mathrm{~s}^{-1}$.

\begin{tabular}{lc}
\hline \hline Method & $k(T)$ \\
\hline MDQT $^{\mathrm{a}}$ & $7.8-13$ \\
VTST/MT $^{\mathrm{b}}$ & $13-16$ \\
QTST $^{\mathrm{c}}$ & $12-17$ \\
QCLE $^{\mathrm{d}}$ & 16 \\
RPMD $^{\mathrm{e}}$ & 1.7 \\
\hline \hline
\end{tabular}

${ }^{a}$ Reference 12 .

${ }^{\mathrm{b}}$ Reference 14

${ }^{\mathrm{c}}$ Reference 16

${ }^{\mathrm{d}}$ Reference 17

${ }^{\mathrm{e}}$ Reference 18

expressions (2.6) and

$$
P_{r}(0)=\operatorname{tr}\left[e^{-\beta \hat{H} / 2}(1-\hat{h}) e^{-\beta \hat{H} / 2}(1-\hat{h})\right] .
$$

The values obtained are $P_{r}(0)=0.88$ and $Q_{p}(T) / Q_{r}(T)$ $=0.51$. This denominator generates a multiplicative correction factor 1.56 for the ratio $C_{f}(t) / Q_{r}(T)$, which is merged in Fig. 4. The resulting quantum-mechanical rate constant is $9.3 \times 10^{10} \mathrm{~s}^{-1}$.

This value represents the first numerically exact quantum mechanical calculation of the rate constant for the AzzouzBorgis model in the framework of the system-bath mapping. In Table III, we compare this result to those of previous studies employing various approximate theories as discussed in the Introduction. It should be noted, though, that these studies have been performed for the original atomistic Azzouz-Borgis model. The rate constants obtained using the molecular dynamics with quantum transitions (MDQT) ${ }^{12}$ approach are in particularly good agreement with that obtained here. Furthermore, it is noteworthy that the variational transition state theory with multi-dimensional tunneling corrections $(\mathrm{VTST} / \mathrm{MT})^{14}$ and in particular the quantum transition state theories (QTST) ${ }^{16}$ are in reasonable agreement with the zerotime limit of the accurate quantum-mechanical flux correlation function calculated using ML-MCTDH. The results of the quantum-classical Liouville equation (QCLE) ${ }^{17}$ are somewhat higher than the value obtained in this work. On the other hand, the recently-obtained ring polymer molecular dynamics (RPMD) ${ }^{18}$ result is significantly lower than the ML-MCTDH and MDQT rate constants. As discussed in Ref. 18, the reasons for this are as yet unclear.

\section{CONCLUDING REMARKS}

In this paper, we have applied a recently proposed approach to accurately evaluate thermal rate constants for chemical reactions in the condensed phase to the Azzouz-Borgis model of proton transfer in a polar solvent. The approach is based on the flux correlation function formalism of reaction dynamics and employs a combination of the ML-MCTDH method for an accurate treatment of quantum dynamics and importance sampling techniques. To apply the methodology to the Azzouz-Borgis model, the atomistic Hamiltonian of this model was mapped within linear response to a system-bath 
model. The results of the accurate ML-MCTDH simulations provided in this work may serve as benchmark for the mapped system-bath model to validate more approximate theories.

We have, furthermore, compared the classical dynamics of the original Azzouz-Borgis model and the mapped system-bath model. Due to the approximations involved in the linear response mapping, the transient dynamics of the flux-correlation function of the two models shows significant differences, although the longer-time dynamics and thus the thermal rate constant is very similar. It is thus important to improve the accuracy of the mapped model in order to provide a better description of the original Azzouz-Borgis Hamiltonian. One possibility to do so is to use a more accurate system-bath model that includes position-dependent friction in order to distinguish the environmental effects on the proton at the transition state from those exerted on the reactants. ${ }^{51,52}$ Thereby the coupling term of the system coordinate to the bath modes will not be linear, but would have a more general functional form. ${ }^{13}$ This will greatly improve the accuracy of the transient dynamics for the mapped model since not only the vicinity of the barrier but also a wide range of the reaction coordinate for proton transfer is included in the linear response mapping. In addition, the relative motion of the proton donor and proton acceptor ("donor-acceptor mode") is known to be highly important in modulating the reaction barrier height and thus the proton transfer rate. This effect may be modeled by including a second degree of freedom in the "system" part of the system-bath model. ${ }^{62}$ The effect of such promoting modes have been found to be of importance also in more complex enzymatic reactions. ${ }^{63}$ Similarly, several more anharmonic intramolecular modes, with more general couplings (i.e., beyond linear coupling) to the bath, may be introduced to model the most important contributions of the "system" to the overall dynamics of proton transfer. It would also be interesting to replace the classical potential of mean force with its quantummechanical counterpart as calculated by path integral molecular dynamics techniques. Along very similar lines, the classical force-force correlation function could be replaced with an approximate quantum-mechanical analog obtained from a ring polymer molecular dynamics ${ }^{64}$ calculation.

It should also be noted that, although the ML-MCTDH method is a numerically exact approach to study timedependent quantum dynamics, for the purpose of describing the quantum dynamics of the original Azzouz-Borgis model, the current ML-MCTDH study on the mapped system-bath model only gives approximate results. This is so because of the approximations involved in the linear response mapping to obtain the system-bath model. It is thus important to exploit the accuracy and feasibility of other dynamical methods, such as those applied previously to the same Azzouz-Borgis model, to treat this important class of problems. Although our ML-MCTDH study does not apply directly to the original Azzouz-Borgis model, it does give accurate quantum dynamics results for the system-bath model described in this paper. Thus, the application of the various approximate methodologies that have been used for the original Azzouz-Borgis problem to the system-bath model will provide an assessment of the accuracy of the approximate dynamical method for treating the proton transfer reaction since the physical regimes de- scribed by the system-bath model is quite similar to that of the original Azzouz-Borgis model. Overall, our accurate MLMCTDH dynamics study on an approximate model Hamiltonian provides a complimentary alternative to other types of study that employ approximate dynamical method to treat more accurate and flexible models. In the long run these two approaches may be further developed and eventually converge to the same accurate result.

\section{ACKNOWLEDGMENTS}

This work has been supported by a Humboldt Research Fellowship from the Alexander von Humboldt Stiftung (I.R.C.), the Deutsche Forschungsgemeinschaft (M.T.), the Fonds der chemischen Industrie (M.T.), and the National Science Foundation CHE-1012479 (H.W.). The calculations were performed at the National Energy Research Scientific Computing Center of US Department of Energy and the Leibniz Rechenzentrum, Munich.

${ }^{1}$ W. H. Miller, S. D. Schwartz, and J. W. Tromp, J. Chem. Phys. 79, 4889 (1983).

${ }^{2}$ T. Yamamoto, J. Chem. Phys. 33, 281 (1960).

${ }^{3}$ H. Wang, D. E. Skinner, and M. Thoss, J. Chem. Phys. 125, 174502 (2006).

${ }^{4}$ N. Makri, Ann. Rev. Phys. Chem. 50, 167 (1999a).

${ }^{5}$ W. H. Miller, Proc. Natl. Acad. Sci. U.S.A. 102, 6660 (2005).

${ }^{6}$ F. Gatti, Theor. Chem. Acc. 116, 60 (2006).

${ }^{7}$ H. Wang and M. Thoss, J. Chem. Phys. 119, 1289 (2003).

${ }^{8} \mathrm{H}$. Wang and M. Thoss, J. Chem. Phys. 131, 024114 (2009).

${ }^{9}$ M. Topaler and N. Makri, J. Chem. Phys. 101, 7500 (1994).

${ }^{10}$ I. R. Craig, M. Thoss, and H. Wang, J. Chem. Phys. 127, 144503 (2007).

${ }^{11}$ H. Azzouz and D. Borgis, J. Chem. Phys. 98, 7361 (1993).

${ }^{12}$ S. Hammes-Schiffer and J. C. Tully, J. Chem. Phys. 101, 4657 (1994).

${ }^{13}$ D. Antoniou and S. D. Schwartz, J. Chem. Phys. 110, 465 (1999).

${ }^{14}$ R. P. McRae, G. K. Schenter, B. C. Garrett, Z. Svetlicic, and D. G. Truhlar, J. Chem. Phys. 115, 8460 (2001).

${ }^{15}$ S. Y. Kim and S. Hammes-Schiffer, J. Chem. Phys. 119, 4389 (2003).

${ }^{16}$ T. Yamamoto and W. H. Miller, J. Chem. Phys. 122, 044106 (2005).

${ }^{17}$ G. Hanna and R. Kapral, J. Chem. Phys. 128, 164520 (2008).

${ }^{18}$ R. Collepardo-Guevara, I. R. Craig, and D. E. Manolopoulos, J. Chem. Phys. 128, 144502 (2008).

${ }^{19}$ J. E. Straub, M. Borkovec, and B. J. Berne, J. Chem. Phys. 89, 4833 (1988).

${ }^{20}$ J. S. Bader, R. A. Kuharski, and D. Chandler, J. Chem. Phys. 93, 230 (1990).

${ }^{21}$ N. Makri, E. Sim, D. E. Makarov, and M. Topaler, Proc. Natl. Acad. Sci. U.S.A. 93, 3926 (1996).

${ }^{22}$ A. O. Caldeira and A. J. Leggett, Ann. Phys. (N.Y.) 149, 374 (1983).

${ }^{23}$ U. Weiss, Quantum Dissipative Systems ( World Scientific, Singapore, 1999).

${ }^{24}$ If the kinetic scheme holds in the long time limit the various correlation functions considered in Ref. 10 give the same rate.

${ }^{25}$ W. H. Miller, J. Phys. Chem. A 102, 793 (1998).

${ }^{26}$ A. N. Drozdov and S. C. Tucker, J. Chem. Phys. 115, 9675 (2001).

${ }^{27}$ D. Chandler, J. Chem. Phys. 68, 2959 (1978).

${ }^{28}$ D. Chandler, Introduction to Modern Statistical Mechanics (Oxford University Press, New York, 1987).

${ }^{29}$ D. Brown and J. C. Light, J. Chem. Phys. 97, 5465 (1992).

${ }^{30}$ U. Manthe, J. Chem. Phys. 102, 9205 (1995).

${ }^{31}$ W. H. Thompson and W. H. Miller, J. Chem. Phys. 106, 142 (1997).

${ }^{32}$ F. Matzkies and U. Manthe, J. Chem. Phys. 106, 2646 (1997).

${ }^{33}$ F. Matzkies and U. Manthe, J. Chem. Phys. 110, 88 (1999).

${ }^{34}$ U. Manthe and F. Huarte-Larranaga, Chem. Phys. Lett. 349, 321 (2001)

${ }^{35}$ S. C. Althorpe and D. C. Clary, Ann. Rev. Phys. Chem. 54, 493 (2003).

${ }^{36}$ M. Thoss, I. Kondov, and H. Wang, Chem. Phys. 304, 169 (2004).

${ }^{37}$ H. Wang and M. Thoss, Chem. Phys. Lett. 389, 43 (2004).

${ }^{38} \mathrm{M}$. Thoss and H. Wang, Chem. Phys. 322, 210 (2006).

${ }^{39}$ I. Kondov, M. Cizek, C. Benesch, H. Wang, and M. Thoss, J. Phys. Chem. C 111, 11970 (2007). 
${ }^{40}$ J. Li, I. Kondov, H. Wang, and M. Thoss, J. Phys. Chem. C 114, 18481 (2010).

${ }^{41}$ U. Manthe, J. Chem. Phys. 128, 164116 (2008).

${ }^{42}$ U. Manthe, J. Chem. Phys. 130, 054109 (2009).

${ }^{43}$ O. Vendrell and H.-D. Meyer, J. Chem. Phys. 134, 044135 (2011).

${ }^{44}$ N. Makri, J. Phys. Chem. B 103, 2823 (1999).

${ }^{45}$ M. P. Allen and D. J. Tildesley, Computer Simulation of Liquids (Oxford Science Publications, Oxford, 1987).

${ }^{46}$ D. Frenkel and B. Smit, Understanding Molecular Simulation - From Algorithms to Applications (Academic, San Diego, 1996).

${ }^{47}$ H. Wang, M. Thoss, and W. H. Miller, J. Chem. Phys. 115, 2979 (2001).

${ }^{48}$ J. P. Bergsma, B. J. Gertner, K. R. Wilson, and J. T. Hynes, J. Chem. Phys. 86, 1356 (1987).

${ }^{49}$ B. J. Gertner, K. R. Wilson, and J. T. Hynes, J. Chem. Phys. 90, 3537 (1989).

${ }^{50}$ J. E. Straub, B. J. Berne, and B. Roux, J. Chem. Phys. 93, 6804 (1990).

${ }^{51}$ B. Carmelli and A. Nitzan, Chem. Phys. Lett. 102, 517 (1983).

${ }^{52}$ G. Haynes and G. Voth, J. Chem. Phys. 103, 10176 (1995).

${ }^{53}$ The energy barrier referred to here is defined as the difference in energy between the reactant minimum and saddle-point transition state of the twodimensional $V_{C}(r, R)$.
${ }^{54}$ J. B. Straus and G. A. Voth, J. Chem. Phys. 96, 5460 (1992).

${ }^{55}$ The chosen constrained value of $\mathrm{r}=1.34 \AA$ corresponds to the position of the barrier in the $\mathrm{R}=2.70 \AA$ cut through the two-dimensional potential of mean force plotted in Fig. 2 of Ref. 16. It is close to the position of the barrier in the one-dimensional potential of mean force $(r=1.30 \AA)$ shown in Fig. 2 of this work.

${ }^{56}$ Using the diatomic reduced mass of A and B.

${ }^{57}$ D. Antoniou and S. D. Schwartz, J. Chem. Phys. 110, 7359 (1999).

${ }^{58}$ C. H. Bennett, in Algorithms for Chemical Computations (American Chemical Society, Washington D.C., 1977).

${ }^{59}$ H. C. Andersen, J. Chem. Phys. 72, 2384 (1980).

${ }^{60}$ I. R. Craig and D. E. Manolopoulos, J. Chem. Phys. 122, 084106 (2005).

${ }^{61}$ W. H. Press, S. A. Teukolsky, W. T. Vetterling, and B. P. Flannery, Numerical Recipes in Fortran 77: The Art of Scientific Computing (Cambridge University Press, Cambridge, England, 1992).

${ }^{62}$ D. Antoniou and S. D. Schwartz, J. Chem. Phys. 108, 3620 (1998).

${ }^{63}$ R. Caratzoulas, D. Antoniou, and S. Schwartz, J. Am. Chem. Soc. 124, 3270 (2002).

${ }^{64}$ I. R. Craig and D. E. Manolopoulos, J. Chem. Phys. 121, 3368 (2004). 